\section{PET Programs for demonstration and student research in cognitive psychology}

\section{GREG B. SIMPSON and CURT BURGESS University of Nebraska, Omaha, Nebraska}

The BASIC programs described here grew partly from a need to create a teaching package for cognitive psychology using the Commodore PET microcomputer. The programs were also designed to be used in an undergraduate laboratory course in human leaming and memory, in which students were required to conduct an experiment of their own design within a single semester. A number of existing commercial packages are available for other small computers (e.g., Fischler, 1980; Keenan \& Keller, 1980). These are excellent for demonstrating a number of research methods in cognitive psychology, but are somewhat limited in their ability to be adapted to run experiments different from those originally demonstrated.

The present package is useful for in-class demonstrations of several experiments widely cited in human memory and cognitive psychology textbooks. Each explains the phenomenon in question, collects data from the subject, and displays a summary of the subject's performance. The programs are written so that they can be adapted easily to student research projects that are similar, but not identical, to those demonstrated. The programs may be modified to generate a large number of experiments based on the four phenomena demonstrated in the original programs.

Each of the demonstration programs begins with a brief description of the research on which the demonstration is based. Three of the programs display a graph showing a typical outcome of the research. The subject then is given instructions and led through a series of sample trials, which may be repeated as necessary. The student then serves as subject in the experiment. With one exception, he/she makes responses directly at the keyboard, and when data collection is complete, a summary of the subject's performance is displayed.

Each of the programs contains REMARK statements indicating the beginning and ending lines of the major sections of the program (description of research, instructions, experiment, and data summary display). In this way, if a user wishes to adapt the program for research using naive subjects, those portions that should be eliminated or changed (such as the description of the research and its results) may be easily identified. In

The authors are grateful to the Committee for the Improvement of Instruction, University of Nebraska at Omaha, for supporting this project. Address correspondence to the first author at the Department of Psychology, University of Nebraska, Omaha, NE 68182. addition, those program statements that set values that users may want to change (e.g., stimulus presentation rates, retention intervals, etc.) are identified by REM statements. A brief description of each of the programs follows.

\section{Description}

Short-Term Memory. This program demonstrates the classic Peterson and Peterson (1959) experiment. On each of 48 trials, the subject sees a consonant trigram for $3 \mathrm{sec}$. He/she then sees a three-digit number and is required to count backward for $3,6,9,12,15$, or $18 \mathrm{sec}$. A recall prompt is given, and the subject enters the trigram at the keyboard. At the end of the experiment, a table displays the percentage of trigrams correctly recalled for each of the six retention intervals.

Short-Term vs. Long-Term Memory. This program represents a partial replication of research by Glanzer and Cunitz (1966), separating the short-term and longterm memory contributions to the typical serial position curve seen in free recall. The subject receives five lists of 20 words each. After each list, a number is presented and the subject counts backward. This is the only program in which the subject makes no repsonses at the keyboard. Requiring the subject to type the words in addition to recalling the items would prove too distracting. Instead, the subject writes the words, each list on a separate sheet of paper. Since the responses are not entered into the computer, there is no way for the program to score and display the subject's performance. Instead, at the completion of the experiment, the five lists are displayed, along with instructions for scoring recall. The demonstration uses a $10-\mathrm{sec}$ retention inter$\mathrm{val}$, with a presentation rate of $6 \mathrm{sec} /$ word. The lines setting these values are identified by REM statements, and, through changes in these lines, a between-subjects replication of all of the conditions used by Glanzer and Cunitz is possible. Generally speaking, this program turns the PET into a memory drum, and may be adapted for any experiment that requires the timed presentation of lists of stimuli.

Scanning Short-Term Memory. This program demonstrates the item-recognition paradigm of Stemberg (1966). On each trial, from one to four digits are presented sequentially. After a 1-sec delay, a probe item is presented, and the subject decides as quickly as possible whether the probe was among the digits presented on that trial. The subject responds "yes" by pressing the "J" key and "no" by pressing the " $F$ " key. A total of 128 trials are presented, 32 of each memory set size. Half of these are positive (yes) trials, and half negative (no). At the completion of the experiment, a table displays the subject's mean reaction time and percentage correct for each of the four memory set sizes (separately for positive and negative trials), and the 
slope and intercept values for the functions relating reaction time to memory set size.

Letter Matching. The final program demonstrates the letter-matching paradigm of Posner, Boies, Eichelman, and Taylor (1969). On each trial, two letters are presented side by side, and the subject makes a speeded decision (again, by pressing the "F" or "J" keys) as to whether the two letters have the same name. There are four stimulus types: physically identical (e.g., AA), same name $(A a)$, same case different $(A B)$, and different case different $(\mathrm{Ab})$. The program also uses three stimulus onset asynchronies (SOAs): 0,750 , and $1,500 \mathrm{msec}$. There are 120 trials, 10 of each stimulus type-SOA combination. Upon completion, a table displays mean reac. tion times and percentages correct for each of the 12 conditions. The program can be easily modified for other SOAs and stimulus types (e.g., words), and can be used for a wide variety of comparison or verification tasks.

\section{Requirements}

The longest of the four programs (memory scanning) requires $28 \mathrm{~K}$ of $\mathrm{RAM}$. If the initial section, which describes the research, is not included, any of the programs is able to run on a $16 \mathrm{~K}$ machine. With their respective descriptions, the memory-scanning and lettermatching programs require at least $32 \mathrm{~K}$. The programs are written in PET BASIC, but can easily be translated to another BASIC dialect.

\section{Limitations}

Because the PET is unable to read both upper- and lowercase letters from DATA statements, stimuli for the letter-matching task are displayed via POKEs to the screen rather than PRINT statements. REM statements make clear which numbers in the DATA statements represent POKEs to the screen and which represent codes for the trial type and SOA for each trial.

The timing for the reaction time experiments is accurate only to the nearest $1 / 60 \mathrm{sec}$, the greatest precision possible with PET's internal clock. The subject's reaction times (in $1 / 60$-sec increments) are then multiplied by 16.67 to provide "millisecond" values for display in the tables. In order to obtain true millisecond timing, a machine language timing loop is required (e.g., Merikle, Cheesman, \& Bray, 1982). However, such a subroutine will not solve the problem if responses are made with the PET keyboard. In order to record a response, the PET checks the memory location that stores the matrix coordinate of a depressed key, to see which key has been pressed. Because such a check is performed every $16.67 \mathrm{msec}$ (Commodore Business Machines, 1979; Donahue \& Enger, 1980), the millisecond value obtained from a machine language timer will be rounded to the nearest $1 / 60 \mathrm{sec}$. Over a large number of trials, the reaction time given will overestimate the true reaction time by an average of $8.3 \mathrm{msec}$. Therefore, either an external response device or clock (or both) is required for true millisecond accuracy. Neither of these alternatives was acceptable in our case because, for ease of using the programs for in-class demonstration, we wanted the system to be completely selfcontained, requiring only the computer and a disk or tape drive. For demonstration and undergraduate research purposes, the $1 / 60$-sec resolution seems to suffice. If either of these programs were to be adapted for more advanced purposes (graduate student or faculty research), they would require an external timer or a machine language subroutine (such as that presented by Merikle et al., 1982) and an external response mechanism (e.g., Burgess \& Furman, 1984).

\section{Availability}

Complete listings of all of the programs, and instructions on their use, may be obtained at no charge from Greg B. Simpson, Department of Psychology, University of Nebraska, Omaha, NE 68182. For purposes of translation to other versions of BASIC, we will also include a note on those statements that are idiosyncratic to the PET.

\section{REFERENCES}

Burgess, C., \& Furman, E. (1984). A microcomputer controlled dual-response device. Unpublished manuscript, Department of Psychology, University of Nebraska, Omaha.

Commodore Business Machines. (1979). CBM 2001-16, -32, 3016, 3032 professional computer user manual (pp. 30-33). Santa Clara, CA: Author.

Donahue, C. S., \& Enger, J. K. (1980). PET-CBM personal computer guide (p. 336). Berkeley, CA: Osborne/McGraw-Hill.

Fischler, I. (1980). An on-line laboratory in cognition and perception. Behavior Research Methods \& Instrumentation, 12, 116-119.

Glanzer, M., \& Cunitz, A. R. (1966). Two storage mechanisms in free recall. Journal of Verbal Learning and Verbal Behavior, 5, 351-360.

Keenan, J. M., \& Keller, R. A. (1980). Teaching cognitive processes: Software for laboratory instruction in memory and cognition. Behavior Research Methods \& Instrumentation, 12, 103-110.

Merikle, P. M., Cheesman, J., \& Bray, J. (1982). PET flasher: A machine language subroutine for timing visual displays and response latencies. Behavior Research Methods \& Instrumentation, 14, 26-28.

Peterson, L. R., \& Pterson, M. J. (1959). Short term retention of individual verbal items. Journal of Experimental Psychology, 58, 193-198.

Posner, M. I., Boies, S. J., Eichelman, W. H., \& Taylor, R. L. (1969). Retention of visual and name codes for single letters. Journal of Experimental Psychology Monographs, 79(1, Pt. 2).

Sternberg, S. (1966). High-speed scanning in human memory. Science, 153, 652-654.

(Manuscript received for publication February 7, 1984.) 